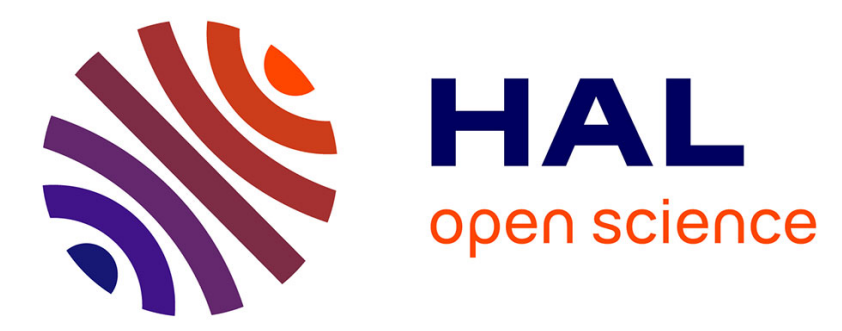

\title{
Satisfaction au travail et culture managériale empathique en EHPAD : étude exploratoire dans deux établissements
}

M. Hergue, P. Lenesley, P. Narme

\section{- To cite this version:}

M. Hergue, P. Lenesley, P. Narme. Satisfaction au travail et culture managériale empathique en EHPAD : étude exploratoire dans deux établissements. NPG: Neurologie - Psychiatrie - Gériatrie, 2019, 19, pp.23 - 29. 10.1016/j.npg.2018.09.004 . hal-03486357

\section{HAL Id: hal-03486357 \\ https://hal.science/hal-03486357}

Submitted on 20 Dec 2021

HAL is a multi-disciplinary open access archive for the deposit and dissemination of scientific research documents, whether they are published or not. The documents may come from teaching and research institutions in France or abroad, or from public or private research centers.
L'archive ouverte pluridisciplinaire HAL, est destinée au dépôt et à la diffusion de documents scientifiques de niveau recherche, publiés ou non, émanant des établissements d'enseignement et de recherche français ou étrangers, des laboratoires publics ou privés.

\section{(ㄷ)(1) $\$$}

Distributed under a Creative Commons Attribution - NonCommerciall 4.0 International 


\title{
SATISFACTION AU TRAVAIL ET CULTURE MANAGÉRIALE EMPATHIQUE EN EHPAD: ÉTUDE EXPLORATOIRE DANS DEUX ÉTABLISSEMENTS
}

\author{
Job satisfaction and empathetic management in nursing homes: An exploratory study comparing two \\ establishments

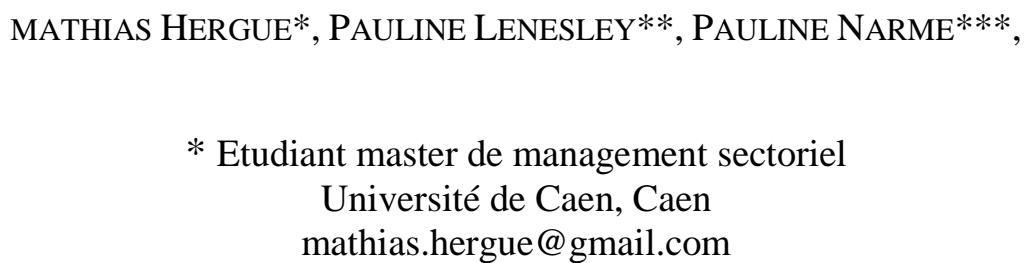 \\ ** Laboratoire COACTIS EA 4161, université de Lyon 2 \\ UFR médecine, Université de Caen, Caen \\ pauline.lenesley@unicaen.fr
*** Laboratoire Mémoire et Cognition, INSERM S894,
Institut de Psychologie
Université Paris Descartes,
Boulogne-Billancourt

\section{$\underline{\text { Auteur correspondant : }}$}

Pauline Narme

Laboratoire Mémoire et Cognition, INSERM S894,

Institut de Psychologie

Université Paris Descartes,

71 avenue Edouard Vaillant,

92100 Boulogne-Billancourt

paulinenarme@gmail.com 


\section{RESUME}

Afin d'étudier les liens entre le style de management et la satisfaction au travail des soignants, une enquête a été menée dans deux EHPAD, le personnel de l'un ayant été formé à la méthode de la Validation $^{\circledR}$. Les objectifs de l'étude étaient de comparer les deux EHPAD quant à (i) la prévalence de l'épuisement professionnel chez les soignants ; (ii) la vision que les soignants avaient de leur hiérarchie (au regard de l'attitude de leur manager).

Les soignants de l'EHPAD ayant été formés à l'attitude empathique (i) présentaient un risque moindre de burnout (aucun d'entre eux ne présentait un risque élevé contre 27\% dans l'autre établissement); avaient une meilleure perception de leur hiérarchie; (iii) décrivaient leurs managers comme ayant une attitude empathique. Cette dernière contribuerait à une meilleure collaboration et favoriserait la satisfaction au travail.

Mots-clés : management ; attitude empathique ; satisfaction au travail ; burnout ; institution.

\section{SUMMARY}

In order to investigate relationships between empathetic style and job satisfaction among nursing staff, an exploratory study was conducted in two different nursing homes. Based on the Validation ${ }^{\circledR}$ method, all staff (including management) were trained in empathy in one nursing home, whereas no training was given in the other. The present study aimed to compare the two nursing homes for (i) burnout prevalence; (ii) nursing staff perceptions of their hierarchy (attitudes of the management).

The nursing staff having received the training (i) presented lower risk of burnout $(0 \%$ had a high risk of burnout versus $27 \%$ in the other nursing home); (ii) indicated a more positive perception of their hierarchy; (iii) were more likely to describe their manager as showing empathy. Empathy could contribute to enhancing collaboration, and consequently to promoting job satisfaction.

Keywords: management; empathetic attitude; work satisfaction; burnout; institution. 
Le paysage français change, le schéma gérontologique évolue, les réformes se succèdent. Les politiques actuelles favorisent le maintien à domicile, notamment dans le cadre du plan gouvernemental «Maladies neurodégénératives »(2014-2019) et dans le cadre de la Silver Economie, proposant diverses solutions pour le Bien-Vieillir. Cependant, le vieillissement entraîne souvent une dépendance telle que le domicile peut devenir inadapté. Lorsque tous les recours, et en particulier les aidants, sont épuisés, l'institutionnalisation devient inévitable. L'entrée en établissement pour personnes âgées dépendantes (EHPAD) est de plus en plus tardive [1] et, de ce fait, le niveau de dépendance plus élevé.

Ce niveau de dépendance alourdit inexorablement la charge de travail des soignants, modifiant ainsi leurs conditions de travail. Les ressources humaines n'ayant peu ou pas augmenté, la priorité est alors donnée aux soins techniques de nursing, délaissant l'accompagnement relationnel. Une certaine pénibilité des tâches apparaît, souvent associée à un manque de connaissances et de reconnaissance, engendrant un risque d'épuisement des soignants ou burnout. Introduit historiquement par Freudenberger [2] en 1974, ce terme a été repris par Maslach [3] pour évoquer « un épuisement mental et physique des personnes dont le travail nécessite un contact permanent avec autrui ». Plus récemment, Schaufeli et Enzmann [4] le décrivent comme " un état d'épuisement physique, émotionnel et mental résultant d'une exposition à des situations de travail émotionnellement exigeantes ». En 2015, l'Institut de veille sanitaire a fait le constat que 480000 personnes sont concernées par la souffrance psychique au travail dont 30000 (toutes professions confondues) présentent un burnout [5]. Dans une étude de l'Observatoire national du suicide réalisée en 2016 [6], parmi les professions les plus touchées, on retrouve les professionnels de la santé et de l'action sociale. Selon l'Institut national de recherche et de sécurité [7], la «facture »du burnout pourrait être estimée entre 2 et 3 milliards d'euros. Les conséquences sont dramatiques pour la santé physique et mentale du salarié [8], pour l'employeur, mais également pour les bénéficiaires du soin (avec un risque accru de maltraitance [9]).

Face à ce constat, il apparaît crucial de mieux comprendre les facteurs qui peuvent contribuer ou, au contraire, prévenir l'apparition du burnout. Si certains facteurs sont directement liés à la difficulté de prendre en soin des personnes présentant des troubles cognitifs et comportementaux, ou le fait d'être fréquemment confronté à la mort [1], d'autres facteurs concernent directement le fonctionnement de la structure et l'organisation du travail [10]. Parmi ces éléments, il apparaît qu'une certaine autonomie [11], un sentiment de reconnaissance et un soutien social de la part de la hiérarchie contribuent grandement à la satisfaction des salariés [12]. Ces aspects peuvent être considérablement influencés par l'attitude 
qu'adoptent la direction et le personnel d'encadrement (cadres de santé), que nous appellerons «managers ». L’impulsion donnée par les managers est essentielle pour pouvoir gérer, piloter et anticiper les différentes situations institutionnelles et permettre aux soignants de pouvoir s'investir pleinement dans la vie de l'établissement. L'attitude adoptée par le manager aura une influence sur l'ensemble de son équipe. Cette attitude peut être [13]: (i) apathique, en l'absence d'émotion, indiquant un état d'indifférence au ressenti de l'autre ; (ii) antipathique, dans le cas d'une aversion aux émotions ou ressentis d'autrui ; (iii) sympathique, lorsque le manager s'implique trop dans les émotions d'autrui, rendant alors limité l'apport de solutions concrètes aux situations difficiles ; ou (iv) empathique, lorsque le manager perçoit le «pathos » de l'autre sans pour autant le partager.

L'empathie peut être définie comme la capacité de ressentir et comprendre l'expérience émotionnelle, mais aussi motrice et somatosensorielle des autres, tout en maintenant consciemment une distinction entre notre propre vécu et celui d'autrui [14]. Une telle attitude pourrait favoriser la communication, la collaboration et le soutien social [15] perçu par les soignants, et par répercussion prévenir l'épuisement professionnel. Bien que peu étudié spécifiquement dans le champ du soin, plusieurs travaux ont montré que la perception que les salariés pouvaient avoir de l'empathie d'un manager permettait à celui-ci une plus grande crédibilité, engendrait davantage de coopération et permettait l'instauration d'un climat de confiance au sein des équipes [16-17]. Ainsi, le bien-être quotidien des employés en serait particulièrement influencé. C'est ce qu'a pu mettre en évidence l'étude de Scott et al [18]. Plus les employés ressentent du soutien social de la part de leur manager, moins ils présentent de plaintes somatiques et plus ils se montrent productifs. De plus, le sentiment d'utilité et de reconnaissance en est valorisé.

La question se pose alors de savoir si une telle attitude empathique peut être développée dans le contexte managérial en EHPAD, notamment grâce à la formation. De plus en plus dispensée dans les établissements médicosociaux, la Validation ${ }^{\circledR}$ est une méthode de communication et de prise en soin qui s'adresse initialement aux aidants ou soignants de personnes atteintes de troubles cognitifs et comportementaux. Développée par Feil [19], cette méthode a pour objectifs de (i) développer une attitude permettant de mieux accompagner les personnes âgées désorientées, (ii) redonner du sens au travail de soignant en gériatrie et (iii) créer de la coopération entre tous les acteurs du soin. Elle repose principalement sur le développement d'une attitude empathique chez les soignants. Toutefois, elle pourrait également permettre de développer l'attitude empathique des managers. 
A ce jour, très peu de travaux se sont intéressés à l'impact de la formation à la méthode de la Validation ${ }^{\circledR}$ sur l'épuisement professionnel des soignants [20] et sur l'attitude managériale, telle qu'elle peut être perçue par les soignants. Les travaux publiés se sont davantage focalisés sur les bénéfices potentiellement observés sur les troubles comportementaux des résidents, montrant d'ailleurs des résultats controversés [21]. Afin d'étudier les liens entre un management lié à l'attitude empathique et la satisfaction au travail des soignants, la présente étude compare la situation dans deux établissements médico-sociaux de type EHPAD, les soignants et les managers de l'un ayant été formés à la méthode de la Validation ${ }^{\circledR}$ contrairement à l'autre. L'objectif principal de cette enquête exploratoire était d'évaluer les signes d'épuisement professionnel, la vision que les soignants avaient des priorités dans leur travail ainsi que leur perception de la hiérarchie, en particulier concernant l'attitude de leur manager et le soutien social. Partant du postulat que la méthode de Validation ${ }^{\circledR}$ favoriserait l'attitude empathique des managers, nous faisions l'hypothèse d'un épuisement professionnel plus faible et d'une perception de la hiérarchie plus favorable dans l'EHPAD dans lequel l'ensemble du personnel avait été formé, comparativement à l'autre EHPAD.

\section{MATERIEL ET METHODE}

\section{Population}

L'enquête a été réalisée auprès de 29 soignants travaillant au sein de deux EHPAD. Pour cela, 20 questionnaires ont été distribués dans chacun des deux établissements : le premier était un établissement privé à but lucratif (EHPAD1), et le second, un établissement public territorial (EHPAD2). A la différence du premier, dans l'EHPAD2, l'ensemble des soignants ainsi que le directeur et les managers (infirmier(ère)s coordinateur(trice)s et référent(e)s, médecins coordonnateurs, adjoint(e)s de direction, et responsable de gestion) ont été formés à l'attitude empathique via la méthode de la Validation®.

Après information sur l'étude et signature d'un consentement éclairé, un questionnaire était donné en mains propres aux soignants avec une enveloppe timbrée pour un retour dans un délai de trois semaines. Quinze questionnaires ont été retournés par l'EHPAD1 contre 14 dans l'EHPAD2 (pourcentage de répondants : respectivement $75 \%$ et $70 \%$ ).

\section{Evaluation de l'épuisement professionnel}


Afin d'évaluer l'épuisement potentiel des soignants, l'échelle Maslach Burnout Inventory (MBI)[22] a été proposée. Cette échelle, composée de 22 affirmations, proposait aux soignants d'évaluer à quelle fréquence ils étaient amenés à avoir certaines pensées (de «jamais » à «chaque jour »). Elle permet d'évaluer les composantes d' «épuisement émotionnel » («Je me sens émotionnellement vidé par mon travail»), de dépersonnalisation («Je sens que je m'occupe de certains patients de façon impersonnelle comme s'ils étaient des objets»), et d'accomplissement personnel («J'ai l'impression à travers mon travail d'avoir une influence positive sur les gens »). Lorsqu'un individu présente des signes de burnout, ses scores d'épuisement émotionnel et de dépersonnalisation sont élevés et son score d'accomplissement diminue. A partir des scores limites proposés par Maslach, des niveaux de burnout (aucun, faible, modéré ou élevé) ont pu être déterminés. Par exemple, un niveau faible correspondait à une seule composante considérée comme élevée d'après les normes, un niveau élevé correspondait à un score élevé sur les trois composantes.

\section{Estimation de la priorité des soignants dans leur travail}

Les soignants étaient ensuite invités à définir leurs priorités dans leur travail. A partir d'une liste de dix réponses possibles (ambiance de travail, autonomie, horaires, hygiène et sécurité, qualité, rémunération, reconnaissance de la hiérarchie, évolution personnelle, formation, reconnaissance de la part des résidents), il leur était demandé de choisir trois réponses et de les classer par ordre de priorité.

\section{Evaluation de la perception des soignants à propos de leur hiérarchie}

Un ensemble de question était ensuite proposé aux soignants. Les cinq premières avaient pour objectifs d'avoir (i) une vision d'ensemble de l'investissement des soignants au sein de leur structure («A quel point connaissez-vous l'organigramme de votre établissement ?»; «A quel point connaissez-vous les points principaux du projet d'établissement?») (ii) ainsi qu'une évaluation subjective de ce que les soignants ressentent de leurs interactions avec leur hiérarchie ( A quel point la hiérarchie vous fait-elle confiance et reconnaît la valeur de votre travail? »; «A quel point votre hiérarchie résout-elle les conflits ?"; "A quel point avez-vous la possibilité de contribuer à l'amélioration du service? »). La cotation allait de $0($ («as du tout ») à 5 (« tout à fait »).

Les trois questions suivantes avaient pour objectif d'évaluer la perception qu'ont les soignants à propos de l'attitude de leur manager («A quel point vous sentez-vous écouté par la hiérarchie?»; 
«...vous sentez-vous compris par la hiérarchie ?»; "A quel point la hiérarchie vous aide-t-elle à évoluer dans votre pratique professionnelle?»). La cotation allait de 0 («pas du tout») à 5 (« tout à fait »). A partir des réponses à ces questions, une attitude comportementale du manager en était déduite. Les réponses 0,1 et 2 ont été catégorisées comme réponse «Non, je ne me sens pas compris par la hiérarchie » et les réponses 3,4 , et 5 catégorisées comme réponse « Oui ». Si la réponse était « Non » aux 3 questions, l'attitude perçue était considérée comme apathique. Au contraire, si la réponse était «Oui » pour les 3 questions, l'attitude était considérée comme empathique. Si, seule la première question donnait lieu à une réponse «Oui », l'attitude était antipathique et, si les deux premières questions donnaient lieu à une réponse « Oui », l'attitude était sympathique [11].

Enfin, quatre questions issues de l'échelle de Karasek [23] étaient proposées afin d'explorer, parmi les risques psychosociaux liés au stress au travail, le facteur «soutien social » relatif au soutien professionnel et émotionnel apporté par la hiérarchie («Mon chef se soucie du bien-être des travailleurs qui sont sous sa supervision»). La cotation allait de 1 («fortement en désaccord ») à 4 (« tout à fait d'accord »). Conformément à la méthodologie proposée par Karasek, les scores à ces quatre questions ont été moyennés.

\section{Analyse des données}

Compte tenu de la taille de l'échantillon et de la distribution des données, des analyses non paramétriques ont été réalisées pour comparer les réponses des soignants de l'EHPAD1 et ceux de l'EHPAD2 (comparaisons inter-groupes) à l'aide du test U de Mann-Whitney. Dans le cas de réponses à choix multiples, une analyse à l'aide d'un $\mathrm{Chi}^{2}$ a permis de comparer la fréquence des réponses données au sein des deux groupes. Enfin, afin d'investiguer le potentiel lien entre l'ancienneté dans la structure et l'épuisement professionnel, des corrélations de Spearman ont été réalisées. L'ensemble des analyses statistiques a été conduit grâce au logiciel SPSS version 18.0 avec un seuil de significativité fixé à $\mathrm{p} \leq 0,05$.

\section{RESULTATS}

\section{Caractéristiques démographiques des répondants}


L'âge moyen des soignants ne différait pas entre les deux structures (EHPAD1 : moyenne = 40,5 ; écart-type $=9,8 ;$ EHPAD2 $\left.: 40,2 \pm 9,4 ; \mathrm{U}_{\text {Mann-Whitney }}=97 ; \mathrm{p}>0,7\right)$. Il existait une surreprésentation de femmes dans l'échantillon, toutefois sans différence statistique entre les deux structures (EHPAD1 : $80 \%$; EHPAD2: $\left.100 \% ; \mathrm{Chi}^{2}{ }_{(1 ; \mathrm{N}=29)}=3,1 ; \mathrm{p}>0,08\right)$. Bien que le nombre d'années d'expérience professionnelle en gériatrie ne différât pas entre les deux structures (EHPAD1 : 9,1 \pm 6,9 ; EHPAD2 : $\left.14,1 \pm 7,6 ; \mathrm{U}_{\text {Mann-Whitney }}=64 ; \mathrm{p}>0,08\right)$, notons que les soignants de l'EHPAD2 présentaient une ancienneté dans l'établissement supérieure $(8,9 \pm 5,6)$ à celle des soignants de l'EHPAD1 $(4,8 \pm 4,4$; $\left.\mathrm{U}_{\text {Mann-Whitney }}=52 ; \mathrm{p}=0,02\right)$.

\section{Composantes de l'épuisement professionnel (MBI)}

La comparaison des scores aux différentes dimensions du MBI a montré que les scores d'épuisement émotionnel sont supérieurs dans l'EHPAD1 $(19,6 \pm 10,8)$ par rapport à ceux recueillis dans l'EHPAD2 $(9,6 \pm 6,1 ; \mathrm{U}=45 ; \mathrm{p}=0,008)$. Il en est de même pour les scores de dépersonnalisation (EHPAD1 : 7,3 \pm 6,5; EHPAD2 : 2,9 $\pm 4 ; \mathrm{U}=51,5 ; \mathrm{p}=0,02)$. En revanche, les deux établissements sont comparables en ce qui concerne les scores d'accomplissement personnel (EHPAD1: 38,9 $\pm 8,9$; EHPAD2 : 41,7 $\pm 6,4 ; \mathrm{U}=95,5 ; \mathrm{p}=0,68)$.

S'il l'on s'intéresse aux différents degrés de burnout, une différence significative est observée quant à la proportion de soignants n'ayant aucun signe de burnout. Celle-ci est significativement plus élevée dans l'établissement $2(71,4 \%)$ comparativement à l'établissement $1\left(33 \% ; \mathrm{Chi}^{2}{ }_{(1 ; \mathrm{N}=29)}=4,2\right.$; $\mathrm{p}=0,04)$. A contrario, la proportion de soignants présentant un degré élevé de burnout est significativement plus élevée dans l'EHPAD1 (26,7\%) que dans l'établissement $2\left(0 \% ; \mathrm{Chi}^{2}{ }_{(1 ; \mathrm{N}=29)}=4,3\right.$ ; $=0,04)$.

\section{Réponses des soignants quant à leur priorité au travail}

Concernant les priorités au travail, seul le premier choix fait par les soignants diffère en fonction de l'EHPAD. Il apparait que, dans l'EHPAD1, la réponse la plus fréquemment sélectionnée concerne l'ambiance de travail (40\% des réponses), tandis que dans l'EHPAD2, les soignants ont dit avoir comme priorité la reconnaissance des résidents (43\%). Cette réponse n'était sélectionnée que dans $7 \%$ des cas dans l'EHPAD1, ce qui constitue une différence significative $\left(\mathrm{Chi}^{2}{ }_{(1 ; \mathrm{N}=29)}=5,2 ; \mathrm{p}=0,02\right)$. 


\section{Perception des soignants de leur hiérarchie}

Il apparaît que les soignants de l'EHPAD2 : (i) connaissent mieux l'organigramme $(4,3 \pm 0,7)$ que ceux de l'EHPAD1 $(2,9 \pm 1,8 ; \mathrm{U}=56 ; \mathrm{p}=0,03)$; (ii) ont tendance à mieux connaître les projets d'établissement $(3,2 \pm 1,1)$ que ceux de l'EHPAD1 $(1,9 \pm 1,8 ; \mathrm{U}=61,5 ; \mathrm{p}=0,057)$; (iii) perçoivent davantage de confiance et de reconnaissance de la part de leur hiérarchie $(4,4 \pm 0,6)$ par rapport à ceux de l'EHPAD1 $(2,7 \pm 2 ; \mathrm{U}=57,5 ; \mathrm{p}=0,03)$; (iv) ont le sentiment que leur hiérarchie résout davantage les conflits $(4,1 \pm 1)$ que ceux de l'EHPAD1 $(1,9 \pm 1,5 ; \mathrm{U}=28 ; \mathrm{p} \leq 0,001)$. Les scores relatifs au sentiment des soignants de pouvoir contribuer à l'amélioration du service ne diffèrent pas significativement (EHPAD2 : 3,8 $\pm 0,6 ;$ EHPAD1 : 2,3 $\pm 2,1 ; \mathrm{U}=68 ; \mathrm{p}=0,11$ ).

Concernant les questions relatives à l'attitude que les soignants perçoivent de leur manager, les résultats montrent que, dans l'EHPAD2, les soignants se sentent (i) davantage écoutés par leur hiérarchie $(4,4 \pm 0,7)$ que dans l'EHPAD1 $(2,2 \pm 1,9 ; \mathrm{U}=34 ; \mathrm{p}=0,001)$; (ii) davantage compris par leur hiérarchie $(4,1 \pm 0,8)$ que ceux de l'EHPAD1 $(2 \pm 1,7 ; \mathrm{U}=28,5 ; \mathrm{p}<0,001)$; (iii) davantage aidés dans l'évolution de leur pratique professionnelle $(4,4 \pm 0,6)$ par rapport à ceux de l'EHPAD1 $(2,1 \pm 1,9 ; \mathrm{U}=38,5 ; \mathrm{p}=0,003)$. Si l'on considère maintenant la catégorisation des réponses à ces mêmes questions en « Oui » / «Non» afin de déduire l'attitude managériale perçue par les soignants, il apparaît que, dans l'EHPAD1, 46,7\% des soignants perçoivent leur hiérarchie comme ayant une attitude apathique, 33,3\% comme ayant une attitude empathique, $13,3 \%$ une attitude sympathique et 6,7\% une attitude antipathique. Au contraire, dans l'EHPAD2, les soignants perçoivent leur hiérarchie comme étant empathique pour 100\% des soignants. La situation est donc significativement différente entre les deux établissements $\left(\mathrm{Chi}^{2}{ }_{(3 ; \mathrm{N}=29)}=\right.$ $14,2 ; \mathrm{p}=0,003)$.

Enfin, concernant le soutien social que les soignants disent percevoir de leur hiérarchie, (sousscore de l'échelle de Karasek), il apparaît que, dans l'EHPAD2, le score moyen de soutien social perçu par les soignants de la part de leur hiérarchie est significativement plus élevé $(3,4 \pm 0,4)$ que celui rapporté par les soignants de l'EHPAD1 $(2,7 \pm 0,6 ; \mathrm{U}=30,5 ; \mathrm{p}=0,001)$.

\section{Etude de corrélation avec l'ancienneté dans la structure}

Afin de vérifier que la différence d'ancienneté dans la structure observée entre les soignants des deux établissements ne contribue pas aux différences observées sur les variables d'intérêt, une étude de corrélation a été conduite entre l'ancienneté des soignants et les scores obtenus au MBI, ainsi qu'avec les 
scores de perception des soignants (de leur hiérarchie, de l'attitude du manager, du soutien social). Aucune corrélation n'était significative entre l'ancienneté et les scores au MBI (tous les p>0,5). Aucune corrélation n'était significative entre l'ancienneté et les scores relatifs à la perception des soignants de leur hiérarchie (tous les p>0,1), excepté leur sentiment de pouvoir contribuer à l'amélioration du service $(\mathrm{r}=0,4 ; \mathrm{p}=0,04)$. Plus l'ancienneté des soignants est importante, plus ils ont le sentiment de pouvoir contribuer à l'amélioration du service.

\section{DISCUSSION}

Ces résultats corroborent notre hypothèse selon laquelle un épuisement professionnel plus faible et une perception de la hiérarchie plus favorable devraient être observés dans l'EHPAD2 comparativement à l'EHPAD1. Premièrement, les scores d'épuisement émotionnel et de dépersonnalisation sont supérieurs dans l'EHPAD1 par rapport à l'EHPAD2. De plus, à partir de l'analyse de ces scores exprimés en degré de burnout, il apparait que la situation est significativement plus favorable dans l'EHPAD2 avec 71,4\% des soignants ne présentant aucun signe de burnout, contre seulement 33\% dans l'EHPAD1. Deuxièmement, le ressenti et la perception des soignants de leur hiérarchie sont bien meilleurs dans l'EHPAD2 que dans l'EHPAD1. Parmi ces indicateurs, la meilleure connaissance de l'organigramme et des projets d'établissement suggère une implication plus importante des soignants au travail dans l'EHPAD2. En effet, selon Lévy-Leboyer [24], ces connaissances engendrent un sentiment d'appartenance qui favorise la satisfaction au travail, l'estime de soi et la productivité au sein des équipes.

Par ailleurs, la perception du soutien social et de la reconnaissance est plus importante dans l'EHPAD2. Ce sentiment pourrait être assimilable à une certaine autonomie accordée par la hiérarchie, qui permettrait de moduler et diminuer le stress des soignants et donc de diminuer l'épuisement professionnel (voir aussi [15] pour une étude en faveur de liens directs entre soutien social et burnout). Cela semble être le cas au vu de l'absence de burnout de degré élevé dans l'EHPAD2.

Enfin, les soignants de l'EHPAD2 décrivent une attitude du manager comme étant plus à l'écoute, les comprenant mieux et les aidant davantage à les faire évoluer dans leur travail que dans l'EHPAD1. Les soignants de l'EHPAD2 ont tous - sans exception - décrit l'attitude de leur manager comme étant empathique, ce qui était le cas pour seulement un tiers des soignants de l'EHPAD2. Ceci indique une différence claire d'attitude du manager entre les deux établissements. Selon Karasek et 
Theorell [11], la qualité de communication - liée au style managérial - est déterminante pour améliorer la satisfaction des salariés au travail. Ces données corroborent les résultats de travaux menés en entreprise, tels que ceux de Scott et al [18] montrant que la satisfaction des salariés était d'autant plus grande que les managers étaient empathiques, tenant alors compte de leurs émotions. Des résultats similaires obtenus dans la population générale ont montré qu'une formation à une «culture empathique » permettait aux individus une meilleure adaptabilité psychologique face aux situations émotionnelles [25]. Ainsi, une attitude empathique du manager favoriserait les ressources émotionnelles du personnel et sa satisfaction professionnelle, et ce pattern se retrouverait - comme le suggèrent nos résultats -, dans le domaine sanitaire et médicosocial. En bonne concordance, une étude récente proposant une formation spécifique à l'empathie à des professionnels du soin a également mis en évidence un bénéfice de telles formations, en contribuant à la prévention de l'épuisement professionnel [26].

Si, de manière générale, les formations du personnel soignant travaillant en EHPAD augmentent significativement leur satisfaction professionnelle et leur engagement dans la structure [27], il est intéressant de noter que nos résultats semblent indiquer que la formation à l'attitude empathique modifie également leurs priorités au travail. En effet, interrogés sur leur priorité, il semble que celle des soignants de l'EHPAD1 soit orientée vers un aspect institutionnel en indiquant comme premier choix l'ambiance de travail. Au contraire, la réponse majoritairement donnée par les soignants de l'EHPAD2 (recevoir la reconnaissance du résident) témoigne d'une priorité orientée vers le bénéficiaire du soin, et suggère le maintien du sens de leur travail.

Bien que les résultats de cette enquête exploratoire aillent dans le sens de nos hypothèses, plusieurs limites méthodologiques doivent être soulignées. Tout d'abord, lors de l'analyse des caractéristiques des répondants, il a été retrouvé une différence significative entre les deux établissements en ce qui concerne l'ancienneté des soignants, les soignants de l'EHPAD2 travaillant dans l'établissement depuis plus longtemps que ceux de l'EHPAD1. Il est alors possible que cette différence contribue à expliquer un burnout moins fréquent chez eux. Toutefois, nous n'avons retrouvé que peu de lien statistique entre ces deux variables. Deuxièmement, en ce qui concerne les deux établissements, certains facteurs qui n'ont pas été mesurés pourraient également contribuer à expliquer les différences observées. Par exemple, il n'a pas été demandé aux soignants s'ils travaillaient dans un secteur particulier (Unité protégée, Pôle d'Activités de Soins Adaptés, etc.) Or, la charge de travail diffère dans ces secteurs en raison des troubles comportementaux pouvant être liés aux syndromes démentiels de certaines personnes 
âgées. De même, des indicateurs tels que le niveau de dépendance moyen ou le niveau de charge moyen en soins techniques n'ont pas été recueillis et peuvent donc différer entre les deux établissements, contribuant également à expliquer une charge de travail et un épuisement consécutif différents. Cette enquête exploratoire justifie néanmoins de poursuivre le travail de recherche dans ce domaine, en établissant - à plus grande échelle - un essai contrôlé comparant deux établissements en ligne de base afin de vérifier qu'ils soient réellement comparables avant toute intervention de formation dans l'un d'entre eux. Enfin, si les dispositifs de formation se développent de plus en plus dans les établissements de soin afin d'améliorer les pratiques des professionnels et la gestion des risques psychosociaux, la recherche actuelle dans ce domaine doit également pouvoir fournir des réponses quant aux types de formation les plus propices à prévenir l'épuisement professionnel. Autrement dit, il apparait indispensable de pouvoir comparer l'efficacité des formations. Ainsi, il reste à confirmer qu'il appartient aux managers de mettre en place des conditions organisationnelles favorables afin de promouvoir le bien-être et la satisfaction des collaborateurs, notamment par le biais d'une attitude empathique.

\section{CONCLUSION}

Le contexte socioéconomique actuel et les conditions de travail en EHPAD, parfois vécues comme difficiles par des personnels très engagés, contribuent à fragiliser et épuiser les équipes soignantes. Dans cet univers où la confrontation à la « grande dépendance » rime souvent avec « demande de performance », le syndrome de burnout est fréquent.

D'après cette étude, la satisfaction des soignants serait d'autant plus importante que les managers dits «empathiques » prennent en compte leurs émotions. Cette capacité managériale apporterait un réel bénéfice sur le bien-être des salariés. Ceux-ci auraient une meilleure perception de leur hiérarchie et lui feraient davantage confiance. Cette confiance vient alimenter les capacités d'autonomisation des collaborateurs, permet à ces derniers d'être encore plus compétents, participatifs et devenir de véritables ressources. Le soutien social est un élément clé de l'attitude empathique. Quand les soignants se sentent écoutés, compris et soutenus, ils se nourrissent d'une dynamique participative qui réduit significativement

le syndrome d'épuisement professionnel et qui augmente leur productivité. La qualité de la communication sera donc importante au sein de l'équipe. Ne serait-il pas essentiel que les managers adoptent une attitude empathique au sein de leur établissement pour veiller au bien-être de leurs 
collaborateurs ? Cette étude tend à montrer qu'une telle attitude semblerait bénéfique et qu'une culture empathique permettrait une meilleure adaptabilité face aux situations émotionnellement lourdes.

Conflits d'intérêt : Les auteurs n'ont pas de conflits d'intérêt à déclarer. 


\section{Références}

1. Marquier R, Vroylandt T, Chenal M, et al. Des conditions de travail en EHPAD vécues comme difficiles par des personnels très engagés. Les Dossiers de la DREES $2016 ; 5: 32$ p.

2. Freudenberger HJ. Staff burn-out. J Soc Issues 1974; 30: 159-65.

3. Maslach C. Burned-out. Hum Behav 1976; 5: 16-22.

4. Schaufeli W, Enzmann D. The burnout companion to study and practice: a critical analysis. London: Taylor and Francis, 1998.

5. Khireddine I, Lemaître A, Homère J, et al. La souffrance psychique en lien avec le travail chez les salariés actifs en France entre 2007 et 2012, à partir du programme MCP. Bull Epidémiol Hebd 2015 ; $23: 431-8$.

6. Observatoire national du suicide. Suicide, connaitre pour prévenir : dimensions nationales, locales et $\begin{array}{llll}\text { associatives. } & 2^{\text {ème }} \quad \text { rapport, } & \text { février } & \text { http://drees.solidarites- }\end{array}$ sante.gouv.fr/IMG/pdf/ons2016_mel_220216.pdf Consulté le 11 septembre 2018.

7. Trontin NC, Lassagne N, Boini S, et al. Le coût du stress professionnel en France en 2007. Institut national de recherche et de sécurité. http://www.inrs.fr/default/dms/inrs/PDF/cout-stressprofessionnel2007.pdf Consulté le 11 septembre 2018.

8. Huguenotte V, Andela M, Truchot D. Développement d'un questionnaire exigences-ressources contextualisé pour une appréhension du burnout des professionnels en EHPAD. Neurol Psychiatr Gériatr 2016; 16(91) : 24-31.

9. Borteyrou X, Paillard E. Burnout et maltraitance chez le personnel soignant en gérontopsychiatrie. Neurol Psychiatr Gériatr $2014 ; 14(81)$ : 169-74.

10. Schaufeli WB, Leiter MP, Maslach C. Burnout: 35 years of research and practice. Career Develop Intern 2009; 14: 204-20.

11. Karasek RA, Theorell T. Healthy work, stress, productivity and the reconstruction of working life. New York: Basic Books, 1990

12. Guéritault-Chalvin V, Cooper C. Mieux comprendre le burnout professionnel et les nouvelles stratégies de prévention: un compte rendu de la littérature. J Ther Comp Cog 2004; 14(2) : 59-70

13. Légeron P. Le stress au travail. Paris : Editions Odile Jacob, 2015.

14. Thirioux B, Mercier MR, Blanke O, et al. The cognitive and neural time course of empathy and sympathy: an electrical neuroimaging study on self-other interaction Neuroscience 2014; 267: 286-306. 
15. Cassel JP. The contribution of the social environment to host resistance. Am J Epidemiol 1976; 104(2): 107-23.

16. George JM. Emotions and leadership: the role of emotional intelligence. Hum Rela 2000; 8: 1027-55.

17. Lewis KM. When leaders display emotion: how followers respond to negative emotional expression of male and female leaders. J Org Behav 2000; 21: 221-34.

18. Scott BA, Colquitt JA, Paddock EL, et al. A daily investigation of the role of manager empathy on employee well-being. Org Behav Hum Dec Process 2010; 113: 127-40.

19. Feil N. Validation : la méthode de Naomi Feil. Pour une vieillesse pleine de sagesse. RueilMalmaison: Editions Lamarre, 2003.

20. Söderlund M, Norberg A, Hansebo G. Validation method training: nurses' experiences and ratings of work climate. Int J Older People Nurs 2014; 9: 79-89.

21. Neal M, Barton Wright P. Validation therapy for dementia. Cochrane Database Syst Rev 2003; 3 : CD001394.

22. Maslach C, Jackson SE. Maslach burnout inventory manual ( $2^{\text {nd }}$ edition). Palo Alto: Consulting psychologists press, 1986.

23. Karasek RA. Job demands, job decision latitude, and mental strain: implications for job redesign. Adm Sci Q 1979; 24: 285-308.

24. Lévy-Leboyer C. Remotiver au travail, développer l'implication de ses collaborateurs. Paris : Editions Eyrolles, 2007.

25. Jazaieri H, McGonigal K, Jinpa T, et al. A randomized controlled trial of compassion cultivation training: Effects on mindfulness, affect, and emotion regulation. Motiv Emot 2013; 38: 23-35.

26. Altmann T, Schönefeld V, Roth M. Evaluation of an empathy training program to prevent emotional maladjustment symptoms in social professions. Psychol 2015; 6: 1893-904.

27. Gurnik M, Hollis-Sawyer L. Empowering assisted living frontline care staffs to better care for Alzheimer's and dementia residents. Ageing Int 2003; 28: 82-97. 